\title{
Investigating possible retinal biomarkers of head trauma in Olympic boxers using optical coherence tomography
}

This article was published in the following Dove Press journal: Eye and Brain

\author{
Charmaine Childs' \\ Lynne A Barker ${ }^{2}$ \\ Alex MD Gage ${ }^{3}$ \\ Mike Loosemore ${ }^{4}$ \\ 'Faculty of Health and Wellbeing, \\ Sheffield Hallam University, Sheffield, \\ South Yorkshire, UK; ${ }^{2}$ Centre for \\ Behavioural Science and Applied \\ Psychology, Sheffield Hallam \\ University, Sheffield, South Yorkshire, \\ UK; ${ }^{3}$ Alex Gage Family Optometrist, \\ Sheffield, South Yorkshire, UK; \\ ${ }^{4}$ Institute of Sport, Exercise and \\ Health (ISEH), London, UK
}

Purpose: Changes to retina have been reported after a number of neurodegenerative conditions. The purpose of this study was to investigate retinal structures in Olympic boxers exposed to frequent head blows.

Methods: Retinal imaging offers potential as a non-invasive biomarker of neuropathology. Macula and retinal nerve fiber layer (RNFL) thickness was measured using optical coherence tomography (OCT) in UK Olympic boxers attending two mandatory eye screening programs, 18 months apart. Data from the two eye screenings provide longitudinal data of retinal change over time. Sedentary healthy subjects (controls) without past or present history of concussion were also screened at the time of the second boxer screening to provide comparison of crosssectional data.

Results: Sixteen Olympic boxers aged 20-33 years and 20 sedentary healthy controls, aged 24-45 years, were recruited. Significant macula thickening was observed over time (18 months) in $75 \%$ of right and $50 \%$ of left eye sectors. For RNFL, left eye quadrants thickened. For right eye RNFL quadrants, thickening and thinning of this layer were observed. Cross-sectional results showed thinner macula sectors and RNFL quadrants in Olympic boxers compared to controls. Conclusion: Significant change to macula and RNFL densities, occurring over an 18 month interval is an unexpected finding in otherwise heathy elite sportsmen. In addition, macula and RNFL were thinner than healthy sedentary controls. OCT may prove clinically useful as a candidate retinal biomarker of neuropathological change after mild traumatic brain injury and/ or repeat head blows.

Keywords: optical coherence tomography, OCT, mild traumatic brain injury, macula, retinal nerve fiber layer, imaging, concussion, eye, biomarkers, chronic traumatic encephalopathy, CTE

\section{Introduction}

Retinal imaging is a well-established, non-invasive technique for capturing eye and systemic disease. The eye and neurosensory retina, as an extension of brain tissue during embryonic development, is a part of the central nervous system and can be visualized relatively easily. Pathological change to eye structures and retinal blood vessels may arguably serve as a proxy of brain change where other imaging techniques (eg, computed tomography, magnetic resonance imaging) are not so readily accessible.

The retinal nerve fiber layer (RNFL) constitutes axonal fibers of retinal ganglion cells (RGC) forming the optic nerve. RNFL conveys visual information to the brain and appears sensitive to pathological brain change. Studies have shown thinning of the RNFL in multiple sclerosis (MS), a degenerative brain condition causing white matter atrophy. ${ }^{1-3}$ Similarly, RNFL and total macular volumes are thinner in Alzheimer's
Faculty of Health and Wellbeing, Sheffield Hallam University, Montgomery House,

32 Collegiate Crescent, Sheffield,

SI02BP, UK

Tel +44 I I4 2252282

Email c.childs@shu.ac.uk 
disease and in Parkinson's disease when comparisons are made with normal ranges, the changes correlating with disease severity. ${ }^{4-6}$

Alterations to the retinal vasculature have also been reported after mild traumatic brain injury (mTBI). Our first in man study showed significantly greater arteriolar and venular tortuosity in patients presenting with two or more postconcussive symptoms after mild to moderate TBI compared to age-, gender-, and race-matched controls. ${ }^{7}$ Beyond these preliminary findings it is presently unclear whether mTBI in humans affects retinal tissue with any correspondence to that which occurs in demyelinating and degenerative conditions. However, this is a plausible consideration given the dual impact of coup, contra-coup acceleration/deceleration forces to the brain. This, together with the secondary damage due to a wave of excitotoxic, metabolic, and inflammatory cascades which follow impact individually contributes to worsening outcome. ${ }^{8,9}$ This combined with disruption to retinal blood supply might deleteriously affect the retina, the most metabolically active tissue of the body. ${ }^{10}$

The clinical consequences of mild to moderate TBI, concussion, and repeated head blows exhibit a change in cognitive function. ${ }^{11}$ Often lingering for weeks and months but without a definite diagnosis, subtle histological, biochemical, and neuronal structural changes evade capture with conventional brain imaging techniques, making it impossible to monitor progressive pathological brain change. In contact sports, repeated head impact occurs frequently, yet little is known as to whether early stage head impact or mild brain trauma is the start of progressive pathological disease processes that could be detected or even halted with screening and surveillance in vivo. For amateur (Olympic) boxers, professional boxers, and for those playing football, rugby, or mixed martial arts, the cumulative effects of brain injury are largely uncharted because quantifying lifetime incidence of brain damage is a major challenge. ${ }^{12}$ Postmortem evaluation is currently the only conclusive method to evaluate cumulative concussive damage to brain and now more usually termed "chronic traumatic encephalopathy" (CTE) of the brain.

CTE was first described in the 1970s based on clinical and neuropsychological features of 15 retired boxers ${ }^{13}$ and more recently has been reported in sportsmen and women exposed to head injuries and in blast-exposed military veterans. ${ }^{14,15}$ CTE may also be influenced by genetic and behavioral factors. ${ }^{14}$ However, common to all known cases of CTE is a history of repeated mild brain trauma with CTE stage correlating with symptom progression. ${ }^{14}$
As initial brain changes in CTE are unremarkable, ${ }^{14}$ they are likely to be overlooked or go undetected with current imaging methods, so there is a pressing need for new in vivo technology to allow monitoring of brain health over lifetime exposure to head trauma (especially for children and youths as they begin to encounter exposure to TBI risk when taking up contact sports). Being able to forewarn of neurocognitive problems and neurodegeneration could have life-long health benefits. Retinal imaging offers one possible solution but data are now needed to show the performance of the technique of OCT for this new application. If changes to retinal parameters could, in the future, fruitfully aid prognosis, management, and treatment when overt brain changes are undetectable by current methods, this technology would reap significant benefits for maintaining brain health in those engaged in contact sports.

This paper represents the first report of OCT in elite athletes (Olympic boxers) where risk of head blows and mTBI would be expected to be greater than in a sedentary group of similarly aged, healthy men and women.

As a start point, the objective of the study was to determine retinal tissue density of presumptive candidate biomarkers, macula, and RNFL, in Olympic boxers compared with healthy controls and to examine whether retinal measurements remain stable or change over time.

\section{Materials and methods}

Institutional ethics approval from Sheffield Hallam University Ethics committee was obtained to access secondary anonymized data of Olympic boxers from those who underwent regular eye screening and vision testing. In the present study, data are presented for two time-points, separated by an interval of 18 months (longitudinal data). As data for Olympic boxers was obtained from a secondary, anonymized source, all boxers who attended the screening sessions were included. For the second screening, only those boxers attending first and second screenings were included to enable "matched" pairs for repeated measures longitudinal analyses. Figures for the number of head blows sustained were not available in this anonymized sample, but an estimation was calculated from number of bouts for the 18-month interval between first and second eye screenings, Time 1 and Time 2, T1 and T2, respectively. With a median of 22 bouts during this period, a typical Olympic Boxer receives approximately eight head blows during a spar session with $35 \%$ of boxers receiving $\geq 10$ head blows. As a conservative estimate, sustained head blows would equate to $\sim 500$ over a 5 -year period based on a 
presumption of five head blows per bout and ten bouts per year over 5 years. Over a period of 18 months to 2 years, this would equate to an estimated 200 head blows. ${ }^{16}$

We recruited sedentary healthy controls not engaged in contact sports to compare with Olympic boxers. Controls responded to an advertisement for the study and were recruited at the time of the second eye screening of Olympic boxers. Participants were included only if there was no known history of concussion or mTBI, systemic illness or eye problems. Participants were excluded if there was known history of diabetes or high myopia. These data of healthy participants provide retinal values for cross-sectional analyses.

\section{Retinal parameters \\ Boxing participants}

Retinal imaging of right and left eyes were performed using a Topcon ${ }^{\mathrm{TM}}$, non-invasive, non-contact, optical coherence tomography (OCT) system (3D OCT 2000, Serial number 673053; Newbury, Berkshire, UK). The system is a nonmydriatic trans-pupillary imaging technique producing high-resolution images of the structure of the retina from the anterior segment to the posterior pole. Images were acquired for mapping of retinal layers, from deep layers (retinal pigment epithelium [RPE]) to RNFL. Data were extracted from scan reports; macula centered report and optic disc centered report, both with $3 \mathrm{D}$ image from $6 \times 6 \mathrm{~mm}$ field.

\section{Non-contact sport controls}

Retinal parameters were acquired for a single retinal scan session using a Topcon ${ }^{\mathrm{TM}}$ Triton, swept source (SS) OCT, deep range imaging (DRI) system (Newbury) (Figure 1). From the optic disc report (3D, $6 \times 6 \mathrm{~mm} ; 512 \times 256$ pixel resolution) and the macula report $(7 \times 7 \mathrm{~mm}, 512 \times 256$ pixel resolution) descriptive data were extracted for retinal parameters, averaged, and used as a comparator for the boxer group. To observe for the effects of raised intraocular pressure (IOP) in controls, measurements were obtained from each eye, at each testing session, using an ocular pressure tonometer (Model TA011; Icare, Vantaa, Finland).

Information from Triton manufacturers' FDA report indicates that measurements taken from both Triton instruments are comparable (https://www.accessdata.fda.gov/cdrh docs/ pdf17/K173119.pdf).

Results are reported for measurements of four outer and inner subfields of the nine subfields on the OCT thickness map defined by the Early Treatment Diabetic Retinopathy Study (EDTRS). ${ }^{17}$ Retinal imaging of right and left eyes were performed using a Topcon ${ }^{\mathrm{TM}}$ (Newbury), non-invasive, non-contact, OCT system. Images were acquired for mapping of retinal layers - from deep layers (RPE) to RNFL. Data obtained from Olympic boxers during the eye screening (performed by $\mathrm{AG}$ ) was transferred via an encrypted hard disk in the form of an Excel (Microsoft ${ }^{\mathrm{TM}}$ ) spreadsheet and without any personal identifiers. OCT values were obtained for macula thickness (microns, $\mu \mathrm{m}$ ) in eight sectors of each eye and for RNFL thickness of optic disc-centered RNFL in four quadrants (superior, inferior, nasal, and temporal). Data were analyzed with SPSS Statistical Package for the Social Sciences (SPSS ver 24, IBM Corporation, Armonk, NY, USA) software. ${ }^{18}$

\section{Results Participants}

Secondary data from 16 Olympic boxers (12 male), mean age 24.5 (SD 3.5, range 20-33) years, attending for two screening sessions $\mathrm{T} 1$ and T2 and 20 healthy, sedentary, adults (eight male), mean age 30.1 (SD 6.2, range 24-45) years, were recruited. Although the control group were older than Olympic boxers, $F(1,34)=7.20, P<0.05$ (because recruitment was from a demographically random selection of interested healthy participants via the university recruitment website), only two participants were outside of Olympic boxers age range (ie, aged 43 and 45 years). The remainder fell within the 24- to 33-year age range of boxers. In the control group, more females were recruited than the Olympic boxer group (12 female controls compared to four females in the Olympic boxer group). We found no significant correlations for age and retinal variables using Pearson's bivariate coefficient. We therefore conducted MANOVAs to compare male with female control data for right and left eye macula sectors and right RNFL and left RNFL quadrants. Using Pillai's trace, no significant effect was observed for gender on left eye macula sectors, $\mathrm{V}=0.59, F(10,9)=1.30, P>0.1$. For right eye macula sectors, Pillai's trace $\mathrm{V}=0.49, F(10,9)$ $=0.88, P>0.1$. For left and right RNFL sectors, Pillai's trace $\mathrm{V}=0.34, F(10,9)=0.47, P>0.1$. Null findings indicated that our control group constituted an appropriate comparator for the Olympic boxer data.

\section{Longitudinal comparison of macula sectors and RNFL quadrants in Olympic boxers at $\mathrm{TI}$ and $\mathrm{T} 2$}

Repeated measures statistics were conducted for right and left eye macula sectors (Tables 1-2) and for right and left eye RNFL quadrants (Tables 3-4) to investigate any potential 

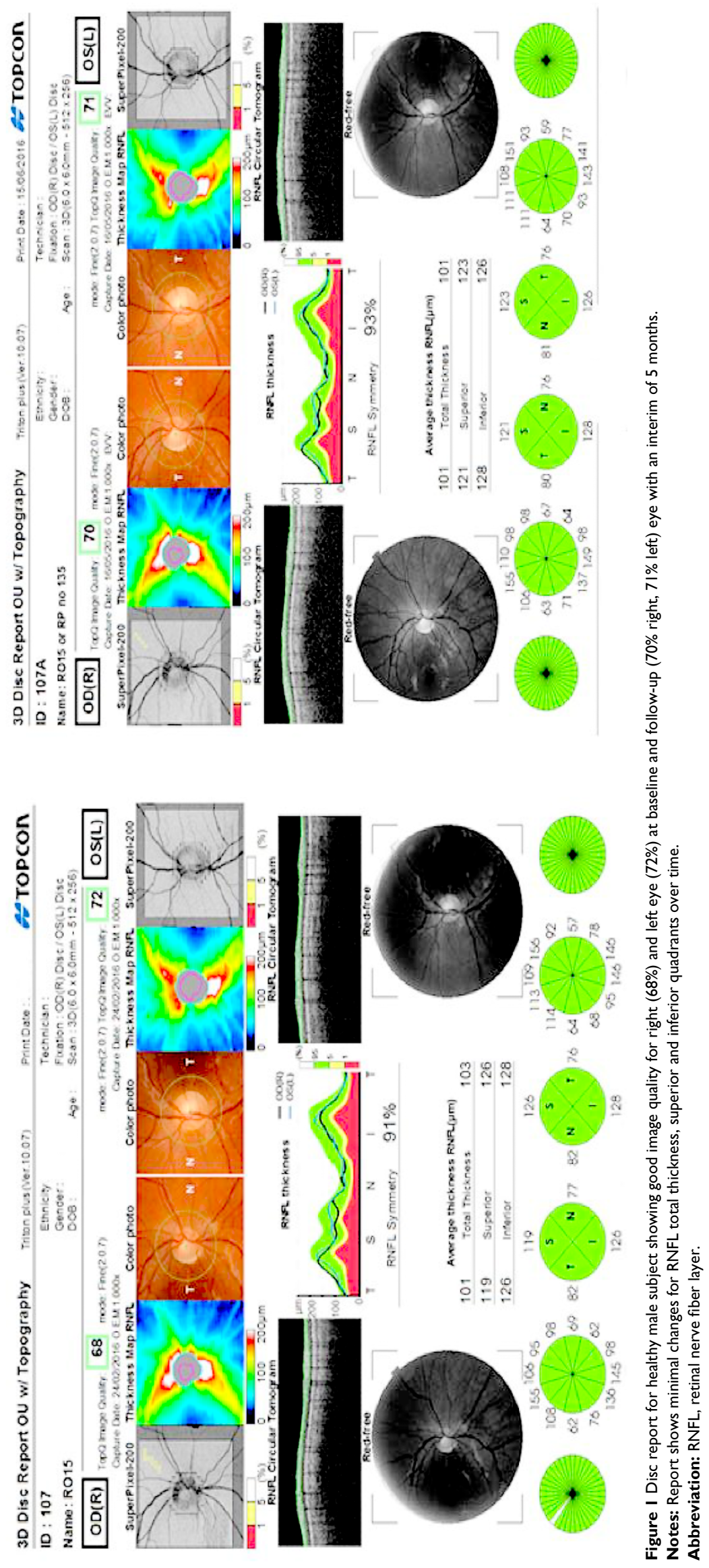
Table I Boxers' ( $\mathrm{N}=16)$ right eye macula data for TI-T2

\begin{tabular}{|l|l|l|l|}
\hline Retinal sector & TI, mean (SE) & T2, mean (SE) & $\begin{array}{l}\text { t-test statistic, two-tailed significance, } \\
\text { and effect size }(\boldsymbol{r})\end{array}$ \\
\hline Inner superior & $305.0(3.2)$ & $3 I 0.5(3.5)$ & $t(I, I 5)=-4.85, P<0.05, r=0.6 I^{\mathrm{a}}$ \\
Inner inferior & $30 I . I(3.3)$ & $303.5(3.6)$ & $t(I, I 5)=-1.0, P>0.05, r=0.0 \mathrm{I}$ \\
Inner nasal & $304.0(4.4)$ & $295.7(3.4)$ & $t(I, I 5)=-2.80, P<0.05, r=0.34$ \\
Inner temporal & $288.3(3.3)$ & $269.9(3.7)$ & $t(I, I 5)=-3.9 I, P<0.05, r=0.5 I^{\mathrm{a}}$ \\
Outer superior & $264.6(3.7)$ & $262.6(4.0)$ & $t(I, I 5)=-3.21, P<0.05, r=0.4 I^{\prime}$ \\
Outer inferior & $256.5(3.1)$ & $286.8(3.8)$ & $t(I, I 5)=-4.21, P<0.05, r=0.54^{\mathrm{a}}$ \\
Outer nasal & $280.6(3.3)$ & $254.0(2.9)$ & $t(I, I 5)=-5.76, P<0.05, r=0.69^{\mathrm{a}}$ \\
Outer temporal & $250.9(3.5)$ & $275.6(3.0)$ & $t(I, I 5)=-1.94, P>0.05, r=0.02$ \\
Average thickness & $270.5(2.9)$ & $7.79(0.8)$ & $t(I, I 5)=-6.84, P<0.05, r=0.76^{\mathrm{a}}$ \\
Total volume & $7.65(0.8)$ & &
\end{tabular}

Notes: ${ }^{a} r$ effects: small $\geq 0.10$, medium $\geq 0.30$, large $\geq 0.50 .{ }^{36}$

Table 2 Boxers' ( $\mathrm{N}=\mathrm{I6})$ left eye macula data for TI-T2

\begin{tabular}{|l|l|l|l|}
\hline Retinal sector & TI, mean (SE) & T2, mean (SE) & $\begin{array}{l}\text { t-test statistic, two-tailed significance, } \\
\text { and effect size }(\boldsymbol{r})\end{array}$ \\
\hline Inner superior & $301.8(4.3)$ & $310.6(3.6)$ & $t(I, I 5)=-2.7 I, P<0.05, r=.33^{\mathrm{a}}$ \\
Inner inferior & $298.5(3.4)$ & $304.4(2.9)$ & $t(I, I 5)=-3.37, P<0.05, r=.43^{\mathrm{a}}$ \\
Inner nasal & $306.2(3.4)$ & $211.7(4.1)$ & $t(I, I 5)=-3.78, P<0.05, r=.49^{\mathrm{a}}$ \\
Inner temporal & $289.2(4.1)$ & $270.3(3.8)$ & $t(I, 15)=-2.90, P<0.05, r=0.36^{\mathrm{a}}$ \\
Outer superior & $264.3(3.9)$ & $262.3(4.1)$ & $t(I, 15)=-2.70, P<0.05, r=0.33^{\mathrm{a}}$ \\
Outer inferior & $257.5(3.2)$ & $285.9(4.0)$ & $t(I, 15)=-2.57, P<0.05, r=0.3 I^{\mathrm{a}}$ \\
Outer nasal & $278.3(3.4)$ & $256.0(3.2)$ & $t(I, 15)=-4.14, P<0.05, r=0.53^{\mathrm{a}}$ \\
Outer temporal & $253.3(3.9)$ & $275.8(3.2)$ & $t(I, 15)=-.956, P>0.05, r=0.20$ \\
Average thickness & $268.3(3.1)$ & $7.77(0.1)$ & $t(I, 15)=-3.70, P<0.05, r=0.47^{\mathrm{a}}$ \\
Total volume & $7.68(0.1)$ & $t(I, 15)=-1.46, P>0.05, r=0.12$ \\
\hline
\end{tabular}

Notes: ${ }^{a} r$ effects: small $\geq 0.10$, medium $\geq 0.30$, large $\geq 0.50 .{ }^{36}$

Table 3 Boxers' $(\mathrm{N}=16)$ right eye RNFL data for TI-T2

\begin{tabular}{|l|l|l|l|}
\hline $\begin{array}{l}\text { Retinal nerve fiber layer } \\
\text { quadrants }\end{array}$ & TI, mean (SE) & T2, mean (SE) & $\begin{array}{l}\boldsymbol{t} \text {-test statistic, two-tailed significance, } \\
\text { and effect size }(\boldsymbol{r})\end{array}$ \\
\hline Superior & $\mathrm{II} 4.5(2.7)$ & $I 20.3(2.7)$ & $t(I, I 5)=-3.42, P<0.05, r=0.44^{\mathrm{a}}$ \\
Inferior & $124.3(2.7)$ & $I 27.3(3.0)$ & $t(I, I 5)=-3.24, P<0.05, r=\left.0.4\right|^{\mathrm{a}}$ \\
Nasal & $88.5(2.1)$ & $79.0(2.6)$ & $t(I, I 5)=7.50, P<0.05, r=0.79^{\mathrm{a}}$ \\
Temporal & $75.8(3.4)$ & $76.0(2.7)$ & $t(I, I 5)=-0.07, P>0.05, r=0.004$ \\
Total average thickness & $101.0(1.6)$ & $100.6(I .6)$ & $t(I, 15)=0.38, P>0.05, r=0.02$ \\
\hline
\end{tabular}

Notes: ${ }^{a} r$ effects: small $\geq 0.10$, medium $\geq 0.30$, large $\geq 0.50 .{ }^{36}$

Abbreviation: RNFL, retinal nerve fiber layer.

Table 4 Boxers' $(\mathrm{N}=16)$ left eye RNFL data for TI-T2

\begin{tabular}{|c|c|c|c|}
\hline $\begin{array}{l}\text { Retinal nerve fiber layer } \\
\text { quadrants }\end{array}$ & TI, mean (SE) & T2, mean (SE) & $\begin{array}{l}\text { t-test statistic, two-tailed significance, } \\
\text { and effect size (r) }\end{array}$ \\
\hline Superior & $118.5(2.1)$ & $119.5(1.8)$ & $t(I, I 5)=-0.45, P>0.05, r=0.01$ \\
\hline Inferior & I27.| (3.5) & | $31.5(3.4)$ & $t(I, 15)=-2.89, P<0.05, r=0.35^{a}$ \\
\hline Nasal $(N=15)^{b}$ & $82.1(2.3)$ & $83.8(2.0)$ & $t(1,14)=-1.83, P>0.05, r=0.19$ \\
\hline Temporal $(\mathrm{N}=15)$ & $70.4(2.2)$ & $67.0(2.1)$ & $t(I, \mid 4)=3.98, P<0.05, r=0.52^{a}$ \\
\hline Total Average Thickness ( $\mathrm{N}=15)$ & $99.8(1.4)$ & $100.2(1.4)$ & $t(I, \mid 4)=-.57, P>0.05, r=0.02$ \\
\hline
\end{tabular}

Notes: ${ }^{a} r$ effects: small $\geq 0.10$, medium $\geq 0.30$, large $\geq 0.50 .{ }^{36}$ bOne participant's data excluded due to outlier.

Abbreviation: RNFL, retinal nerve fiber layer. 
longitudinal change to retinal layers. Tables 1 and 2 show the mean values and standard errors (SEs), inferential statistics, significance, and effect size for each eye sector and quadrant. No prediction was made about potential direction of findings, so conservative two-tailed significance is reported throughout.

Six of eight macula sectors of the right eye (Table 1) were consistently (shown by the direction of $t$-value) and significantly thicker at the T2 screening (18 months from T1) of the Olympic boxers. Large effect sizes (above 0.5) indicate that the results are unlikely due to chance. For the right eye, inner inferior and outer temporal sectors, and for the left eye, outer temporal and total volume did not differ significantly over time.

For the left eye (Table 2), results show that seven of eight macula sectors of the left eye were (shown by the direction of $t$-value) significantly thicker at second screening and with moderate effect sizes. This compares with the large effect sizes observed for the right eye. It is worth noting here that it is difficult to interpret this pattern of findings in this, the first study, but results may reflect the pattern and target of the blows to the head (ie, that one side of the head received more blows and this in turn affects the integrity of retinal structures of that eye more than the other eye).

\section{RNFL}

Results of analyses for right eye RNFL quadrants showed that superior and inferior quadrants were significantly thicker at second screening, while the nasal quadrant was significantly thinner (Table 3). The large effect size for the nasal quadrant analysis indicates that this finding is robust and unlikely to be attributable to error. There were no changes to temporal quadrant and total average thickness. Left eye RNFL descriptive data and analyses are shown in Table 4.
Results of analyses for left RNFL sectors showed that the inferior quadrant was significantly thicker and temporal quadrant was significantly thinner at second screening. (Inferior quadrant was also significantly thicker in right eye RNFL data.) Again, significant findings had moderate to large effect sizes. The pattern of both thinning and thickening seems specific to RNFL, whereas results for macula showed only thickening over time.

\section{Cross-sectional analyses: boxers and controls at T2}

Comparisons were made for macula and RNFL thickness between Olympic boxers and controls. Controls were tested during the months that the boxers' second eye screening was undertaken.

\section{Macula}

Independent MANOVA with group (Olympic boxer and control) as between-subjects factor, and right eye macular variables as dependent variables found (Pillai's trace) a significant effect of group on right eye macula variables for the boxers and controls, $\mathrm{V}=0.99, F(11,24)=13.23, P<0.05$. Separate univariate ANOVA on outcome variables revealed significant as well as non-significant differences between groups for right eye macula variables. The conventional effect size calculations for ANOVA $\left(\eta^{2}\right)$ were computed for all outcome analyses (Table 5).

Control right eye macula thickness measurements were significantly greater than Olympic boxer measurements for most sectors (Table 5). Inner superior, inner nasal, outer inferior, and outer nasal values were higher for controls compared to the boxers but not significantly so. All other sectors were significantly different with small to medium effect size (Table 5). Overall, for controls, macula density

Table 5 Right eye macula descriptive data, ANOVA post hoc results, and $\eta^{2}$ effect size for boxers and controls

\begin{tabular}{|l|l|l|l|}
\hline Retinal sector & $\begin{array}{l}\text { Boxers T2, mean } \\
(\mathbf{S E}), \mathbf{n = 1 6}\end{array}$ & $\begin{array}{l}\text { Control T2, mean } \\
(\mathbf{S E}), \mathbf{n = 2 0}\end{array}$ & $\begin{array}{l}\text { F-test statistic, two-tailed significance, } \\
\text { and } \boldsymbol{\eta}^{2} \text { effect size }\end{array}$ \\
\hline Inner superior & $310.5(3.5)$ & $318.8(3.2)$ & $F(I, 34)=3.00, P>0.05, \eta^{2}=0.08$ \\
\hline Inner inferior & $303.5(3.5)$ & $3 I 6.2(3.4)$ & $F(I, 34)=6.57, P<0.05, \eta^{2}=0.16^{\mathrm{a}}$ \\
\hline Inner nasal & $311.4(3.5)$ & $319.9(3.8)$ & $F(I, 34)=2.59, P>0.05, \eta^{2}=0.07$ \\
\hline Inner temporal & $295.7(3.4)$ & $306.3(3.4)$ & $F(I, 34)=4.6 I, P<0.05, \eta^{2}=0.12^{\mathrm{a}}$ \\
\hline Outer superior & $269.9(3.7)$ & $279.0(2.3)$ & $F(I, 34)=4.62, P<0.05, \eta^{2}=0.12^{\mathrm{a}}$ \\
\hline Outer inferior & $262.6(4.0)$ & $268.3(2.6)$ & $F(I, 34)=I .48, P>0.05, \eta^{2}=0.04$ \\
\hline Outer nasal & $286.8(3.8)$ & $296.1(3.0)$ & $F(I, 34)=3.75, P>0.05, \eta^{2}=0.09$ \\
\hline Outer temporal & $254.0(2.9)$ & $263.0(2.4)$ & $F(I, 34)=5.75, P<0.05, \eta^{2}=0.14^{\mathrm{a}}$ \\
\hline Average thickness & $275.6(3.0)$ & $284.2(2.4)$ & $F(I, 34)=4.60, P<0.05, \eta^{2}=0.1 I^{\mathrm{a}}$ \\
\hline Total volume & $7.79(0.8)$ & $8.03(0.7)$ & $F(I, 34)=4.60, P<0.05, \eta^{2}=0.1 I^{\mathrm{a}}$ \\
\hline
\end{tabular}

Notes: aSmall to medium effect size. 
was thicker than Olympic boxers for right eye, whether the difference was significant or not.

Left eye macula data were similarly analyzed with group as between-subjects factor and left eye macula variables as dependent variables. There was a significant effect of group on left eye macula variables, Pillai's trace $\mathrm{V}=0.85, F(11,24)$ $=12.43, P<0.05$. Separate univariate ANOVA on outcome variables revealed significant and non-significant differences between groups for left eye macula (Table 6), as found for right eye sectors.

Once again, control left eye macula measurements were consistently higher than Olympic boxer measurements but not significant for inner nasal, outer inferior, outer nasal, outer temporal, and average thickness. All other sectors were significantly different with small to medium effect size (Table 6). Thus the same pattern of thicker macula sectors for controls compared to the boxers was found.

On removal of one outlier (Table 6), total average thickness was significantly different for Olympic boxers and controls $F(1,33)=3.93, P<0.05$.

Overall, findings showed that the control group macula measurements were greater than the Olympic boxer measurements for both left and right eyes.

\section{RNFL}

There was a significant effect of group on RNFL left/right eye measurements for Olympic boxers and controls, Pillai's trace $\mathrm{V}=0.76, F(11,24)=7.04, P<0.05$. Post hoc ANOVA showed significant and non-significant differences between the boxers and the controls.
For right eye, ANOVA revealed significant differences in two RNFL quadrants (Olympic boxers vs controls): inferior, mean $=127.3($ SE 3.5) vs 139.2 $($ SE 3.1), $F(1,34)=6.31$, $P<0.05, \eta^{2}=0.16$; nasal, mean $=79.0$ (SE 3.2) vs 89.0 (SE $2.8), F(1,34)=5.52, P<0.05, \eta^{2}=0.14$; and for total right eye average thickness mean $=100.6$ (SE 1.8) vs 106.4 (SE 1.6), $F(1,34),=5.65, P<0.05, \eta^{2}=0.14$. Control RNFL quadrant measurements were significantly greater than the boxer measurements indicating a thicker RNFL layer.

For left eye, once again, ANOVA revealed significant differences in one RNFL quadrant (Olympic boxers vs controls): superior, mean $=120.3(\mathrm{SE} 2.7)$ vs 123.1 (SE 2.4), $F(1,34)$ $=8.44, P<0.05, \eta^{2}=0.11$, total volume mean $=7.8(\mathrm{SE} 0.09) \mathrm{vs}$ 8.0 (SE 0.07), $F(1,34)=4.22, P<0.05, \eta^{2}=0.11$, and for total average thickness mean $=100.6(\mathrm{SE} 1.8)$ vs $106.4(\mathrm{SE} 1.6), F$ $(1,34)=5.45 p<0.05, \eta^{2}=0.14$ with higher values for controls.

\section{Summary}

The results of these data analyses showed that Olympic boxers undergoing eye screening at two time points, 18 months apart, had thicker macula density by sector for both eyes and thicker (as well as thinner) RNFL quadrants. Comparison of crosssectional data measurements at the $\mathrm{T} 2$ time-point showed that the Olympic boxers' macula and RNFL measurements were lower compared to controls, despite the significant changes to retinal measurements shown in the Olympic boxers over the 18-month period.

\section{Discussion}

This is a novel study exploring the possibility that OCT may provide a candidate method to capture retinal biomarkers

Table 6 Left eye macula descriptive data, ANOVA post hoc results, and $\eta^{2}$ effect size for boxers and controls

\begin{tabular}{|l|l|l|l|}
\hline Retinal sector & $\begin{array}{l}\text { Boxers T2, mean } \\
\text { (SE) } \mathbf{n = 1 6}\end{array}$ & $\begin{array}{l}\text { Control T2, mean } \\
\text { (SE) } \mathbf{n = 2 0}\end{array}$ & $\begin{array}{l}\text { F-test statistic, two-tailed significance, } \\
\text { and } \boldsymbol{\eta}^{2} \text { effect size }\end{array}$ \\
\hline Inner superior & $310.7(3.6)$ & $320.7(2.9)$ & $F(I, 34)=4.65, P<0.05, \eta^{2}=0.12^{\mathrm{a}}$ \\
Inner inferior & $304.4(2.9)$ & $316.2(3.2)$ & $F(I, 34)=6.96, P<0.05, \eta^{2}=0.17^{\mathrm{a}}$ \\
Inner nasal & $311.7(4.2)$ & $320.6(3.6)$ & $F(I, 34)=2.61, P>0.05, \eta^{2}=0.07$ \\
Inner temporal & $294.5(3.1)$ & $306.4(3.3)$ & $F(I, 34)=6.39, P<0.05, \eta^{2}=0.15^{\mathrm{a}}$ \\
Outer superior & $270.3(3.8)$ & $279.8(2.1)$ & $F(I, 34)=5.30, P<0.05, \eta^{2}=0.13^{\mathrm{a}}$ \\
Outer inferior & $262.3(4.1)$ & $267.3(2.7)$ & $F(I, 34)=1.08, P>0.05, \eta^{2}=0.03$ \\
Outer nasal & $285.9(4.0)$ & $291.3(5.1)$ & $F(I, 34)=0.62, P>0.05, \eta^{2}=0.01$ \\
Outer temporal & $256.0(3.2)$ & $262.5(2.6)$ & $F(I, 34)=2.48, P>0.05, \eta^{2}=0.06$ \\
Average thickness & $275.8(3.2)$ & $297.8(I 2.3)^{\mathrm{b}}$ & $F(I, 34)=2.40, P>0.05, \eta^{2}=0.06$ \\
Total volume & $7.77(0.1)$ & $8.0(0.1)$ & $F(I, 34)=4.42, P<0.05, \eta^{2}=0.1 I^{\mathrm{a}}$ \\
\hline
\end{tabular}

Notes: a'Small to medium effect size. 'O One participant's data excluded due to outlier. A measurement of 529.6 for average thickness in the control group skewed the data to generate a large standard error of 12.3 . 
of brain injury. In this study, macula thickness and RNFL thickness values were investigated in a young, fit, and healthy population of Olympic boxers and healthy non-contact controls. As a cohort, this group of elite sportsmen and women are exposed to risk of TBI, as evidenced by the number of bouts (median 22) over the 18 months of this study. However, at this stage of our investigations, our interpretation of results are limited by lack of exact data on frequency of head blows or sub-concussive events, during competition and during training (sparring), where it is expected that there would be an additional exposure risk.

Six right eye and seven left eye macula sectors out of a total of eight on the EDTRS map were significantly thicker in young, healthy, Olympic boxers over an 18-month period with moderate to large effect sizes for all analyses. Effect sizes are a standardized and objective measure of effect magnitude and indicate that statistically significant findings are robust. An increase in macula measurements (thickening) in young healthy Olympic boxers on repeat testing over an 18-month period using the same retinal imaging equipment and administered by the same examiner has several possible interpretations. First, there is a possibility of diurnal variation in overall retinal thickness of between $5 \%$ and $10 \%$ as suggested by clinical opinion. Second, in our cohort the complex picture of change in measurement values is of interest, potentially reflecting pathological influences but not consistently so. For example, changes were observed in some sectors but not others, there were different sector and quadrant thickness changes to each eye, as well as thinning and thickening of RNFL fibers from screening at $\mathrm{T} 1$ and $\mathrm{T} 2$. This casts doubt on a diurnal variation explanation as the pattern is inconsistent. Indeed, Sharifipour et al (2016) have shown no diurnal variation in a number of measured retinal parameters. ${ }^{19}$ In addition, it is now known that choroid and RPE volumes are the retinal layers most sensitive to diurnal fluctuations, ${ }^{20}$ but as these layers were not included in the study measurements, the changes we have observed are unlikely to be explained by diurnal variation in macula and RNFL thickness. Another possibility is that the presence of microcystic macula edema (MME), which is not condition-specific, but may nevertheless be an early sign of optic neuropathy, might contribute to eventual thinning of $\mathrm{RNFL}^{21}$ as reported by Abegg et al, but MME was not detected in this series. It might be plausible that increased retinal vessel permeability or impaired lymphatic drainage might influence the retinal measurements of the boxers and, since we have previously shown an effect of TBI on retinal vessel parameters (tortuosity) this would be an area of future investigation. ${ }^{7}$
At the present time the mechanisms of retinal change after human mTBI remain poorly understood so few solid conclusions can be drawn on potential pathological causes beyond the effects commensurate with repeated head trauma. Furthermore, retinal changes in the Olympic boxers from T1 to T2 were opposite to what might be expected from the literature of neurodegenerative conditions $;{ }^{1-6}$ rather than a thinning of macula and/or RNFL, we report thickening over time.

With respect to RNFL, right eye superior and right eye inferior plus left eye inferior quadrants were significantly thicker at the second screening but right eye nasal quadrant and left eye temporal quadrant were significantly thinner at the second screening. Nasal and temporal quadrant are typically the thinnest RNFLs in health when measured using Triton instruments (SS or SD systems; https://www. accessdata.fda.gov/cdrh docs/pdf17/K173119.pdf) and we posit the most likely affected by head trauma/head blows as we report here. Again as for macula changes, this pattern of findings is not easily explained by diurnal variation. The specificity of thinning to right eye nasal and left eye temporal quadrants seem to align with a biomechanical account of purported change, particularly since side-to-side head jarring is common in boxers during head blows. There is an extensive literature on RNFL thinning in other degenerative and demyelinating conditions ${ }^{1-6}$ and thinning also occurs naturally with age. ${ }^{21}$ By contrast, far less is known about how mTBI might impact upon retinal thickness in man, ${ }^{22}$ although there is a growing body of evidence investigating traumatic optic neuropathy (TON) after brain injury. ${ }^{23-27}$ Here, indirect TON is specifically associated with concussion but is difficult to detect through normal eye examination particularly in the early stages. ${ }^{23-27}$ There is also evidence that RNFL and RGC layers become thin around 2 weeks after $\mathrm{mTBI}$ in those with indirect TON and that this continues up to 20 -weeks post-trauma. Visual defects however are not always present. ${ }^{28}$

In murine models of brain injury there is evidence of selective pathological effects of mTBI on optic tract that is not easily detected on histological examination. Evenson et a ${ }^{29}$ found myelin injury, gliosis, and axonal degeneration to optic tract. The authors concluded that axons from RGCs (RNFL), but not RGC bodies, were directly injured by mTBI causing thinning. Özevren and Deveci ${ }^{30}$ showed that experimentally induced blast injury maintained over three successive days produced a selective inflammatory response to retina. Other studies in neuro-ophthalmology show inflammation, glial responses, and neuronal loss to retina at 30 -days post-trauma, with no indication of cognitive dysfunction on tests or visible 
pathology to frontal brain regions. ${ }^{31}$ Animal models therefore provide insights into sequelae of blast-induced head trauma and initiation of retinal pathology, but at the present time there is insufficient human data to determine which mechanism is responsible for the changes we have observed in Olympic boxers studied over an interval of 18 months.

The only explanation that we are aware of for RNFL thickening is edema possibly associated with anterior optic neuritis. ${ }^{32}$ In addition, it is unclear from the longitudinal data alone whether macula and RNFL thickness levels differ significantly from expected densities. It is for this reason that we undertook to compare Olympic boxer retinal measurements with healthy controls. Although the controls were slightly older, sedentary ("non-sporty") individuals with no past or current history of being engaged in contact sports and neither a history of TBI nor concussion, we found that overall, macula sectors and RNFL quadrants were thinner in the boxers, even where those differences were not significant. OCT values for healthy eyes, without glaucoma, lose $\sim 0.017 \%$ density per year; an overall loss of 10-20 $\mu \mathrm{m}$ (micron) over a 60-year period. ${ }^{33}$ A significant loss of thickness, especially of macula, would not be expected in otherwise fit and healthy young adults. If our data in Olympic boxers represent a true loss of retinal tissue thickness, over and above expected loss for this age group it is entirely plausible that these data reflect a pathological process not previously reported. Nevertheless, this work should stimulate further international enquiry as to the lasting effects of mTBI for those engaged in all levels of contact sports; a concern for brain health currently attracting intense media interest.

We acknowledge a number of limitations with the study; the first being the retrospective nature of the boxer data. This meant that due to anonymized data, it was not possible to investigate precise contributory factors that might offer further explanation for the changes reported here (ie, history of concussion and head blows). In addition, with the variety of OCT systems available on the market, it is appreciated within the industry that variations in values for thickness of retinal layers, as well as reproducibility of measurements, will have some impact on results. ${ }^{34}$ For example, with each new development in OCT technology, even for the same manufacturer, segmentation algorithms, software, and the operator will have an impact on measurement. For the OCT systems used in this study, this may well have a bearing on our findings. The differences reported in RNFL thickness for example, between Olympic boxers at the two screenings, using the same OCT system and performed by the same operator are significantly different at three quadrants (right eye) and two quadrants (left eye), yet the values are different by up to $6 \mu \mathrm{m}$ only. That these differences are due to variability in measurement cannot be ruled out although moderate to large effect sizes reported here would not be expected in findings caused by measurement error.

Of note is a recent publication ${ }^{35}$ reporting significant differences of 4-6 $\mu \mathrm{m}$ in RNFL thickness between collision sports athletes and controls using spectral domain OCT. In the current study of Olympic boxers, the retinal variable values at the second screening when compared with sedentary controls showed greater differences for macula (both eyes) but also differences of up to $12 \mu \mathrm{m}$ were observed for right eye RNFL. Leong et al's (2018) study found a similar order of thinning in a small cohort of boxers with an average of $10.8 \mu \mathrm{m}$ of thinning compared to controls accompanied by vision-based changes. ${ }^{35}$ It would seem that while difference in the OCT systems (SD vs SS) in the current study could have had a bearing on results due to segmentation effects, it is of interest that patterns of RNFL "thinning" are consistent across the studies. Combined together these data might catalyze explorations of retinal structures in contact sports using OCT to identify biomarkers of repeated mild brain trauma in the same way that retinal biomarkers currently signify neurodegenerative conditions.

\section{Conclusion}

In this study data are presented for a first-step investigation of possible macula and RNFL biomarkers of mTBI in Olympic boxers exposed to repeated head trauma. Limitations include use of secondary data but a deliberate step to protect the anonymity of the boxers. It is also recognized that there is a lack of precise visual, behavioral, and concussive indices. Such limitations will be addressed in our ongoing studies. Our findings and the data of others when considered together suggest that OCT of the retina opens up a novel pathway to the effects of mild TBI on brain via detailed imaging of the neurosensory tissue of the eye.

\section{Acknowledgments}

We thank Megan McTiffin for her assistance in collecting retinal data for controls, Dr Martin McKibbin, Tom Willis and Mike Stockton for assistance with preparation of the Boxer database, and we thank the participants in this study.

\section{Author contributions}

All authors contributed to data analysis, drafting and revising the article, gave final approval of the version to be published, and agree to be accountable for all aspects of the work. 


\section{Disclosure}

The authors report no conflicts of interest in this work.

\section{References}

1. Frohman EM, Fujimoto JG, Frohman TC, Calabresi PA, Cutter G, Balcer LJ. Optical coherence tomography: a window into the mechanisms of multiple sclerosis. Nat Clin Pract Neurol. 2008;4(12):664-675.

2. Parisi V, Manni G, Spadaro M, et al. Correlation between morphological and functional retinal impairment in multiple sclerosis patients. Invest Ophthalmol Vis Sci. 1999;40(11):2520-2527.

3. Fisher JB, Jacobs DA, Markowitz CE, et al. Relation of visual function to retinal nerve fiber layer thickness in multiple sclerosis. Ophthalmology. 2006;113(2):324-332.

4. Iseri PK, Altinaş O, Tokay T, Yüksel N. Relationship between cognitive impairment and retinal morphological and visual functional abnormalities in Alzheimer disease. J Neuroophthalmol. 2006;26(1):18-24.

5. Berisha F, Feke GT, Trempe CL, McMeel JW, Schepens CL. Retinal abnormalities in early Alzheimer's disease. Invest Ophthalmol Vis Sci. 2007;48(5):2285-2289.

6. Bodis-Wollner I. Retinopathy in parkinson disease. J Neural Transm. 2009;116(11):1493-1501.

7. Childs C, Ong YT, Zu MM, Aung PW, Cheung CY, Kuan WS. Retinal imaging: a first report of the retinal microvasculature in acute mild traumatic brain injury. Eur J Emerg Med. 2014;21(5):388-389.

8. Lozano D, Gonzales-Portillo GS, Acosta S, et al. Neuroinflammatory responses to traumatic brain injury: etiology, clinical consequences, and therapeutic opportunities. Neuropsychiatr Dis Treat. 2015;11(11):97-106.

9. Denniss R, Barker LA, Francis J, Day C. The Effect of Micronutrient Interventions on Cognitive Rehabilitation in TBI Patients. London, UK: LSNeuron Conference; February 2016.

10. Léveillard T, Sahel JA. Metabolic and redox signaling in the retina. Cell Mol Life Sci. 2017;74(20):3649-3665.

11. Galetta KM, Barrett J, Allen M, et al. The King-Devick test as a determinant of head trauma and concussion in boxers and MMA fighters. Neurology. 2011;76(17):1456-1462.

12. Bernick $\mathrm{C}$, Banks $\mathrm{S}$. What boxing tells us about repetitive head trauma and the brain. Alzheimers Res Ther. 2013;5(3):23.

13. Corsellis JA, Bruton CJ, Freeman-Browne D. The aftermath of boxing. Psychol Med. 1973;3(3):270-303.

14. Stein TD, Alvarez VE, McKee AC. Chronic traumatic encephalopathy: a spectrum of neuropathological changes following repetitive brain trauma in athletes and military personnel. Alzheimers Res Ther. 2014;6(1):4.

15. Mckee AC, Robinson ME. Military-related traumatic brain injury and neurodegeneration. Alzheimers Dement. 2014;10(3 Suppl):S242-S253.

16. Matser EJ, Kessels AG, Lezak MD, Troost J, Jordan BD. Acute traumatic brain injury in amateur boxing. Phys Sportsmed. 2000;28(1):87-92.

17. Early Treatment Diabetic Retinopathy Study Research Group. Photocoagulation for diabetic macular edema. Report number 1. Arch Ophthalmol. 1985;103:1796-1806.
18. IBM Corp. IBM SPSS Statistics for Windows, Version 24.0. Armonk, NY: IBM Corp; 2016.

19. Sharifipour F, Farrahi F, Moghaddasi A, Idani A, Yaseri M. Diurnal variations in intraocular pressure, central corneal thickness, and macular and retinal nerve fiber layer thickness in diabetics and normal individuals. J Ophthalmic Vis Res. 2016;11(1):42-47.

20. Mrejen S, Spaide RF. Optical coherence tomography: imaging of the choroid and beyond. Surv Ophthalmol. 2013;58(5):387-429.

21. Abegg M, Dysli M, Wolf S. Microcystic Macular Edema. Retrograde maculopathy caused by optic neuropathy. Opthalmol. 2014;121:142-149.

22. Hondur G, Göktaş E, Al-Aswad L, Tezel G. Age-related changes in the peripheral retinal nerve fiber layer thickness. Clin Ophthalmol. 2018;12: 401-409.

23. Sarkies N. Traumatic optic neuropathy. Eye. 2004;18(11):1122-1125.

24. Singman EL, Daphalapurkar N, White H, et al. Indirect traumatic optic neuropathy. Mil Med Res. 2016;3(1):2.

25. Ellis MJ, Ritchie L, Cordingley D, Essig M, Mansouri B. Traumatic optic neuropathy: a potentially unrecognized diagnosis after sports-related concussion. Curr Sports Med Rep. 2016;15(1):27-32.

26. Jang SY. Traumatic optic neuropathy. Korean J Neurotrauma. 2018;14(1):1-5.

27. Rossetto JD, Novais E, Capó H. Evolution of optic nerve and retina alterations in a child with indirect traumatic neuropathy as assessed by optical coherence tomography. Arq Bras Oftalmol. 2017;80(6):390-392.

28. Kanamori A, Nakamura M, Yamada Y, Negi A. Longitudinal study of retinal nerve fiber layer thickness and ganglion cell complex in traumatic optic neuropathy. Arch Ophthalmol. 2012;130(8):1067-1069.

29. Evanson NK, Guilhaume-Correa F, Herman JP, Goodman MD. Optic tract injury after closed head traumatic brain injury in mice: a model of indirect traumatic optic neuropathy. PLoS One. 2018;13(5): e0197346.

30. Özevren H, Deveci E. Effects of Potentilla fulgens on the changes made in the retinal damage induced by traumatic head injury. Int J Morphol. 2017:35:840-844.

31. Mammadova $\mathrm{N}$, Ghaisas $\mathrm{S}$, Zenitsky G, et al. Lasting retinal injury in a mouse model of blast-induced trauma. Am J Pathol. 2017;187(7): 1459-1472.

32. Rebolleda G, Diez-Alvarez L, Casado A, et al. OCT: New perspectives in neuro-ophthalmology. Saudi J Ophthalmol. 2015;29(1):9-25.

33. Kanamori A, Escano MF, Eno A, et al. Evaluation of the effect of aging on retinal nerve fiber layer thickness measured by optical coherence tomography. Ophthalmologica. 2003;217(4):273-278.

34. Pierro L, Gagliardi M, Iuliano L, Ambrosi A, Bandello F. Retinal nerve fiber layer thickness reproducibility using seven different OCT instruments. Invest Ophthalmol Vis Sci. 2012;53(9):5912-5920.

35. Leong D, Morettin C, Messner LV, et al. Visual structure and function in collision sport athletes. J Neuroophthalmol. 2018;38(3):285-291.

36. Cohen J. Statistical Power Analysis for the Behavioral Sciences. 2nd edition. Hillsdale, NJ: Lawrence Earlbaum Associates; 1988.
Eye and Brain

\section{Publish your work in this journal}

Eye and Brain is an international, peer-reviewed, open access journal focusing on clinical and experimental research in the field of neuroophthalmology. All aspects of patient care are addressed within the journal as well as basic research. Papers covering original research, basic science, clinical and epidemiological studies, reviews and evaluations, Submit your manuscript here: https://www.dovepress.com/eye-and-brain-journal guidelines, expert opinion and commentary, case reports and extended reports are welcome. The manuscript management system is completely online and includes a very quick and fair peer-review system, which is all easy to use. Visit http://www.dovepress.com/testimonials.php to read real quotes from published authors. 